\title{
Bloqueio do Ramo Esquerdo Idiopático e Sintomas Inexplicáveis Durante o Exercício: Um Relato de Caso
}

\author{
Idiopathic Left-Bundle Branch Block and Unexplained Symptom At Exercise: A Case Report
}

Guilherme Veiga Guimarães ${ }^{1}$ e Edimar Alcides Bocchi ${ }^{1}$

Universidade de São Paulo Instituto do Coração, ${ }^{1}$ São Paulo, SP - Brasil

\section{Introdução}

A presença de bloqueio do ramo esquerdo (BRE) na aparente ausência de outra doença cardíaca suscita questões e preocupações a respeito da estratificação de risco de eventos ou sintomas cardiovasculares subsequentes..$^{1-5}$ Estima-se que a detecção de BRE em adultos assintomáticos, inclusive em atletas, varie entre $0,1 \%$ e $0,8 \%$, o que muito provavelmente corresponde a doenças cardíacas estruturais, e não a respostas fisiológicas ao exercício. ${ }^{6-8}$ Por outro lado, alguns estudos demonstraram que o risco de morte dos pacientes com BRE e doença cardíaca varia entre $2,4 \%$ e $11 \%$ ao ano. ${ }^{9}$

Embora vários estudos tenham sugerido que o BRE induzido por exercício esteja geralmente associado à doença cardiovascular, sobretudo à doença arterial coronariana, há estudos contrastantes que demonstram uma relação entre o BRE induzido por exercício e artérias coronárias normais. ${ }^{6,7,9}$ Entretanto, os efeitos cardiovasculares adversos relacionados ao exercício no BRE com função cardíaca normal em repouso ainda são pouco definidos.

Este relato de caso examinou a relação entre o exercício, o BRE, sintomas e capacidade de exercício em mulher mais jovem com BRE típico, sem história de doença cardiovascular, que relatou ansiedade e falta de ar súbitas durante exercício vigoroso, o que pode ser sugestivo de doença cardíaca, tendo sido encaminhada para teste de esforço.

\section{Relato de Caso}

Mulher saudável de 42 anos de idade com BRE, que relatou ansiedade e falta de ar súbitas durante exercício vigoroso, encaminhada para teste cardiopulmonar do exercício (TCPE) para avaliação dos sintomas inexplicáveis. Ela não estava tomando nenhuma medicação e não tinha histórico médico relevante. Sem sintomas prévios sugestivos de doença cardíaca (desconforto no peito, palpitações, desmaios e angina). Não

\section{Palavras-chave}

Bloqueio de Ramo; Exercício; Atividade Física; Consumo de Oxigênio/fisiologia; Doenças Cardiovasculares/prevenção e controle.

Correspondência: Guilherme Veiga Guimarães •

Universidade de São Paulo Instituto do Coração - Av. Dr. Eneas de Carvalho

Aguiar, 44. CEP 05403-000, São Paulo, SP - Brasil

E-mail: gvguima@usp.br

Artigo recebido em 05/06/2019, revisado em 25/07/2019,

aceito em 10/09/2019

DOI: https://doi.org/10.36660/abc.20190363 havia história de doença neuromuscular ou pulmonar. Ela não fumava nem consumia álcool. Não havia história familiar de doença cardíaca ou ataque cardíaco. Nos seis meses anteriores, ela vinha se exercitando três vezes por semana em uma academia. O programa de exercício consistia em sessões de pelo menos 60 minutos de atividade regular de intensidade moderada, incluindo exercícios aeróbicos, de fortalecimento e flexibilidade muscular e de fortalecimento do equilíbrio. Seu exame físico foi considerado normal, o IMC era $21,5 \mathrm{~kg} / \mathrm{m}^{2}$ e a pressão arterial em repouso estava em 110/70 $\mathrm{mmHg}$. O eletrocardiograma (ECG) em repouso mostrou ritmo sinusal (RS) e frequência cardíaca ( $F C$ ) de 70 bpm, com característica dominante de bloqueio intraventricular: complexo QRS prolongado $(\geq 0,12 \mathrm{~s})$ resultante do atraso na ativação do ventrículo esquerdo, acompanhado de morfologia característica do complexo QRS.6 A angiotomografia coronariana (ATC) foi realizada, e não mostrou depósitos de cálcio e gordura nas artérias coronárias, nem artérias coronárias com estenose. O hemograma completo mostrou resultados normais: glicemia de jejum: $78 \mathrm{mg} / \mathrm{dL}$; colesterol lipoproteína de baixa densidade (LDL): $168 \mathrm{mg} / \mathrm{dL}$; colesterol lipoproteína de alta densidade (HDL): $81 \mathrm{mg} / \mathrm{dL}$; colesterol total (CT): $159 \mathrm{mg} /$ dL; lipoproteína (a) [Lp(a)]: 7 mg/dL; triglicérides (Tg): 49 mg/dL e creatina fosfoquinase (CPK): $26 \mathrm{U} / \mathrm{L}$. A ressonância magnética (RM) do coração mostrou função biventricular normal, fração de ejeção do VE de $65 \%$ e dimensões preservadas, exceto por um movimento septal anormal.

Foi submetida ao TCPE em uma esteira. Ao longo do TCPE, o ECG de 12 derivações mostrou ritmo sinusal (Figura 1). As aferições da pressão arterial estavam dentro da normalidade: em repouso (126/82 $\mathrm{mmHg}$ ), pico (160/90 $\mathrm{mmHg}$ ) e recuperação (120/90 mmHg). Ela parou o exercício por causa da fadiga ( $R E R=1,29)$. Os valores de pico de consumo de oxigênio $\left(\mathrm{VO}_{2}\right.$, pico $\left.=27,1 \mathrm{ml} / \mathrm{kg} / \mathrm{min}\right)$ e frequência cardíaca máxima ( $\mathrm{FCM}=176 \mathrm{bpm}$ ) obtidos no TCPE foram normais para a idade e o sexo: 95\% e 102\%, respectivamente (https:// www.ahajournals.org/doi/10.1161/01.CIR.91.2.580). Do estágio 14 do protocolo de Balke modificado até o final do teste, o TCPE identificou uma redução do $\mathrm{VO}_{2}$ e do pulso $\mathrm{O}_{2}\left(\mathrm{VO}_{2} / \mathrm{FC}, \mathrm{ml} / \mathrm{bpm}\right)$, e um aumento da $\mathrm{FC}$ e da relação espaço morto/volume corrente $(\mathrm{Vd} / \mathrm{Vt}$ ) (Figura 2). A partir deste evento, a inclinação da relação entre ventilação minuto/ produção de dióxido de carbono $\left(\mathrm{VE} / \mathrm{VCO}_{2}\right)$ aumentou abruptamente e não foi acompanhada de hipóxia (Figura 2).

\section{Discussão}

Tanto quanto é do nosso conhecimento, relatamos pela primeira vez o caso de uma paciente mais jovem com BRE sem cardiomiopatia aparente e com sintomas inexplicáveis 


\section{A}

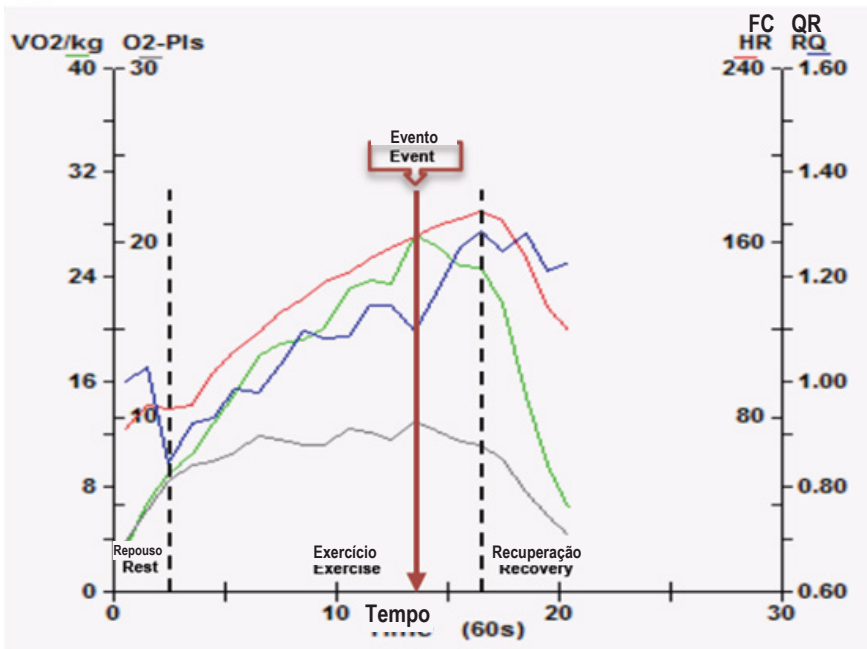

B

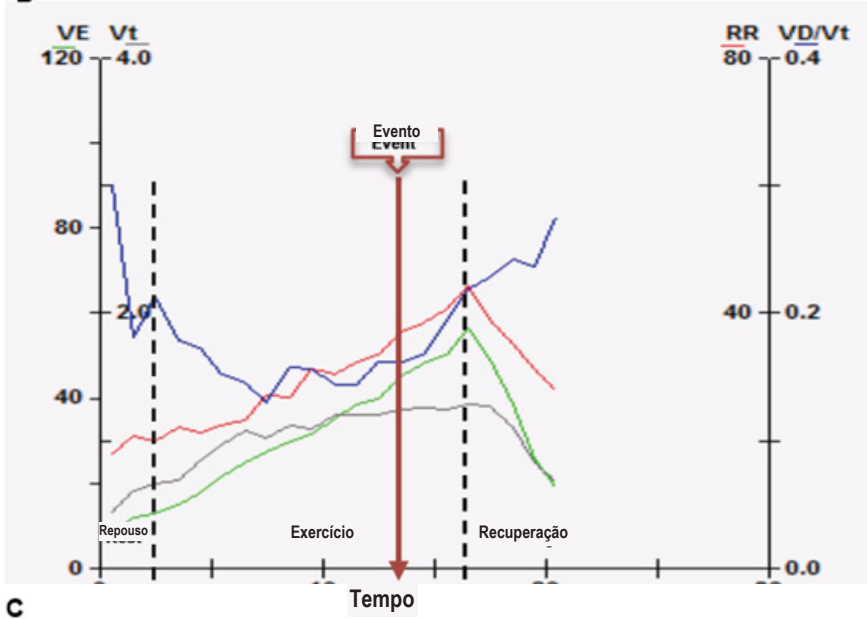

C

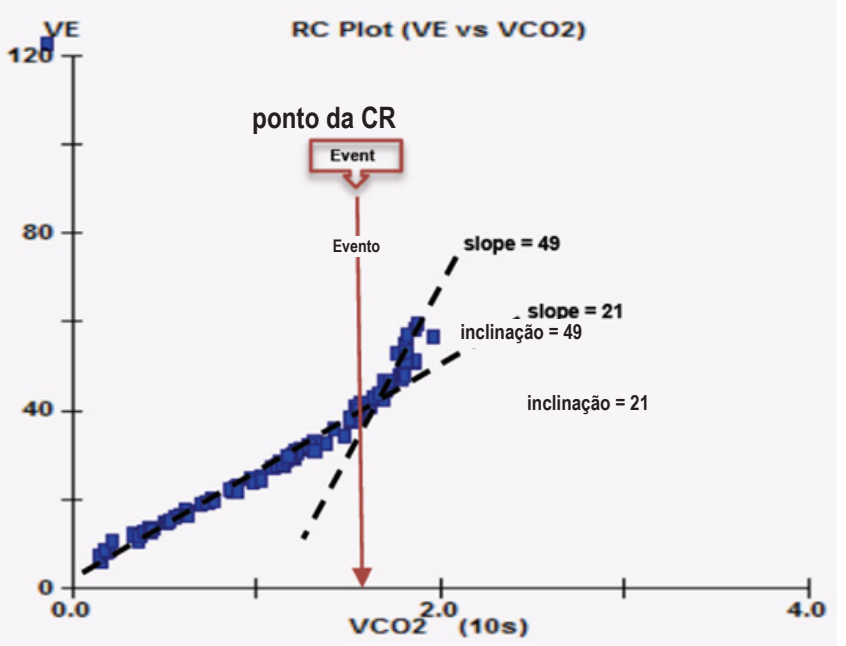

Figura 1 - Teste cardiopulmonar do exercicio em repouso, durante o exercício e a recuperação. As linhas pretas indicam a transição entre essas fases. A) linha verde - consumo de oxigênio (VO/. $\mathrm{kg}, \mathrm{ml} / \mathrm{kg} / \mathrm{min})$, linha vermelha - frequência cardiaca ( $F C$, bpm), linha preta - pulso de oxigênio $\left(\mathrm{O}_{2}-\mathrm{Pls}, \mathrm{m} / \mathrm{bpm}\right.$ ) e linha azul - quociente respiratório (QR); B) linha verde - ventilação por minuto (VE, l/min), linha vermelha - taxa respiratória (RR), linha preta - volume corrente (Vt, $\mathrm{ml} / \mathrm{min}$ ) e linha azul - razão espaço morto/volume corrente (VdNt). C) Inclinação da relação entre ventilação minuto/produção de dióxido de carbono (VENCO). Na primeira parte do exercício, a inclinação VENCO ${ }_{2}$ está normal (21); a partir do momento do evento, durante o teste de esforço, a inclinação $\mathrm{VENCO}{ }_{2}$ aumentou vertiginosamente (49). Seta marrom - momento do evento. 


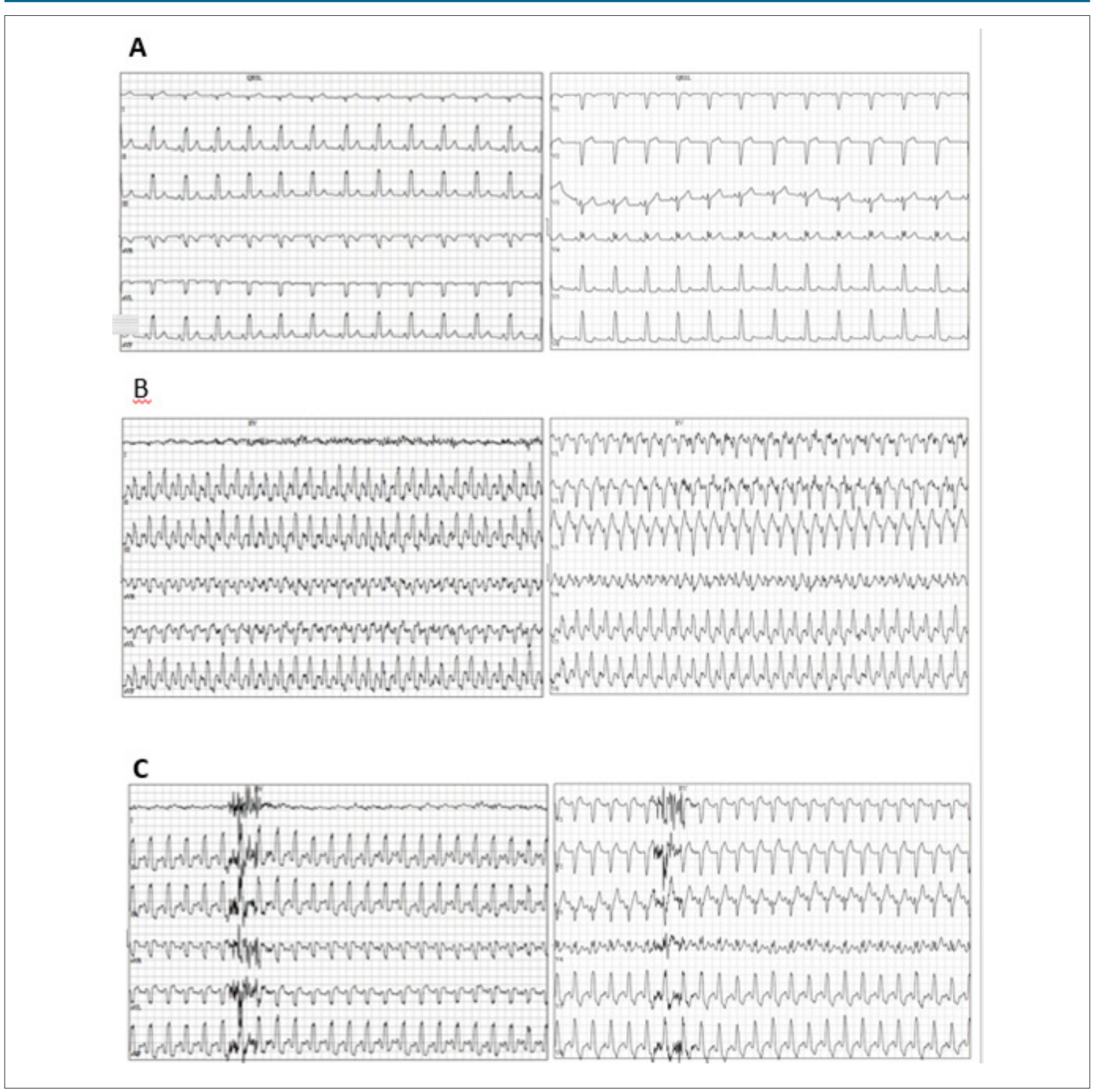

Figura 2 - Eletrocardiograma demonstrando bloqueio de ramo esquerdo: taxa de repouso de 75 bpm (A), frequência cardiaca de 175 bpm durante o teste de tolerância ao exercício máximo (B) e frequência cardiaca de 153 bpm no primeiro minuto do período de recuperação (C).

durante exercício vigoroso (redução no $\mathrm{VO}_{2}$ durante o TCPE), o que é sugestivo de função cardíaca comprometida como consequência de estresse cardiovascular. O ECG não revelou anomalias, a não ser pelo BRE. A reserva de frequência cardíaca e a pressão arterial permaneceram normais ao longo do TCPE.

O exercício oferece uma ferramenta útil para a avaliar indiretamente a reserva funcional cardíaca, a partir do desempenho ventricular esquerdo, as alterações do repouso para o pico do esforço, podendo ser limitado na presença de doenças. Nesse contexto, uma queda no padrão cardiovascular de resposta do $\mathrm{VO}_{2}$ pode indicar um comprometimento do débito cardíaco (DC) ou da extração periférica de oxigênio, conforme observado na insuficiência cardíaca.10 Por outro lado, nos indivíduos saudáveis, o nível de aumento do consumo e extração periférica de oxigênio em resposta ao exercício é muito maior, quando comparado com as alterações no volume sistólico, e semelhante ao aumento observado na FC.

A redução do $\mathrm{VO}_{2}$ e do pulso $\mathrm{O}_{2}$, apesar do aumento na FC, observada nesse caso, pode indicar uma possível anomalia cardíaca. Sugerimos que o movimento assíncrono do ventrículo esquerdo, com o atraso da contração de suas paredes, pode diminuir a carga de trabalho do ventrículo esquerdo, resultando em um volume sistólico mais baixo e indicando uma diminuição do débito cardíaco durante o exercício máximo, apesar do 
aumento na FC. ${ }^{8-10}$ Essa redução na carga de trabalho do ventrículo esquerdo por um aparente defeito de perfusão septal durante o TCPE pode ocasionar perda de energia e "desperdício" de trabalho miocárdico, o que pode representar o impacto hemodinâmico da ativação elétrica assíncrona do miocárdio no BRE, ${ }^{8-10}$ além de explicar, em parte, a queda do $\mathrm{VO}_{2}$ observada durante o TCPE. Por outro lado, o bloqueio do ramo esquerdo induzido por exercício pode estar relacionado ou não a alterações cardíacas aparentes. Entretanto, os pacientes com este achado apresentaram taxas de mortalidade por todas as causas significativamente maiores se comparados àqueles sem bloqueio do ramo esquerdo induzido por exercício. ${ }^{11}$

Essa aparente diminuição da função cardíaca durante o exercício pode ser decorrente de uma diminuição transitória do volume sistólico, provavelmente relacionada a uma piora da função ventricular esquerda (VE), associada a um aumento repentino da inclinação $\mathrm{VE} / \mathrm{VCO}_{2}$, dando origem a uma maior razão entre o espaço morto e o volume corrente $(\mathrm{Vd} / \mathrm{Vt})$ e a um aumento precoce da frequência respiratória, como mecanismos compensatórios. 1,3,5,10 Esse distúrbio na fisiopatologia está relacionado com a disfunção do VE, provocada ou piorada pelo BRE, o que pode ocasionar indiretamente uma disfunção ventricular direita, por meio do aumento da pressão de enchimento do lado esquerdo, causando alterações na função das vias aéreas dos pulmões, e o surgimento de troca anormal de gases em decorrência da disfunção alvéolo-capilar. ${ }^{3,4}$ Além disso, uma inclinação VE/VCO 2 mais elevada é indicativa de hipertensão pulmonar secundária, como consequência de outras condições primárias, tais como insuficiência cardíaca ou doença pulmonar. ${ }^{3,4}$

\section{Referências}

1. Belli JFC, Bacal F, Bocchi EA, Guimaras GV. "Ergoreflex activity in heart failure." Arq Bras Cardiol.2011;97(2):171-8.

2. Breithardt G, Breithardt OL. Left bundle branch block, an old-new entity. J Cardiovasc Transl Res.2012;5(2):107-16.

3. Farina S, Correale M, Bruno N, Paolillo S, Salvioni E, Badagliacca R, et al., Stefania, et al. The role of cardiopulmonary exercise tests in pulmonary arterial hypertension."Eur Respir Rev. 2018;27(148):17034.

4. Guazzi M, Cahalin L, Arena R. Cardiopulmonary exercise testing as a diagnostic tool for the detection of left-sided pulmonary hypertension in heart failure. J Card Fail. 2013;19(7):461-7.

5. Guimarães GV, Belli JC, Bacal F, Bocchi EA. Comportamento dos quimiorreflexos central e periférico na insuficiência cardíaca. Arq Bras Cardiol. 2011; 96(2):161-7.

6. Kim JH, Baggish A L. Electrocardiographic right and left bundle branch block patterns in athletes: prevalence, pathology, and clinical significance. J Electrocardiogr.2015;48(3):380-4.

\section{Consideração}

O teste cardiopulmonar do exercício no BRE, na ausência de outras doenças cardíacas, deve ser considerado como uma técnica para avaliar a capacidade de exercício em pacientes com sintomas inexplicáveis.

\section{Contribuição dos Autores}

Concepção e desenho da pesquisa, Obtenção de dados e Revisão crítica do manuscrito quanto ao conteúdo intelectual importante: Guimarães GV; Análise e interpretação dos dados e Redação do manuscrito: Guimarães GV, Bocchi EA.

\section{Potencial Conflito de Interesses}

Declaro não haver conflito de interesses pertinentes.

\section{Fontes de Financiamento}

Guimarães GV foi financiado pelo Conselho Nacional de Desenvolvimento Científico e Tecnológico (CNPq: 301957/2017-7).

\section{Vinculação Acadêmica}

Não há vinculação deste estudo a programas de pósgraduação.

\section{Aprovação Ética e Consentimento Informado}

Este artigo não contém estudos com humanos ou animais realizados por nenhum dos autores.

7. Kim JH, Baggish AL. Significance of electrocardiographic right bundle branch block in trained athletes. Am J Cardiol. 2011;107(7):1083-9.

8. Koepfli P, Wyss C, Gaemperli O, Siegrist PT, Klainuti M, Schepis T, et al. "Left bundle branch block causes relative but not absolute septal underperfusion during exercise. Eur Heart J. 2009;30(24): 2993-9.

9. Lamberti M, Ratti G, Di Miscio G, Franciolini E. Cardiovascular risk in young workers with left bundle branch block. Open J Prev Med. 2014;4(5):270-4.

10. Lim HS, Hoong S, Theodosiou M. Exercise ventilatory parameters for the diagnosis of reactive pulmonary hypertension in patients with heart failure. J Card Fail. 2014;20(9):650-7.

11. Stein R, Ho M, Oliveira CM, Ribeiro JP, Lata K, Abella J, et al. Exerciseinduced left bundle branch block: prevalence and prognosis. Arq Bras Cardiol.2011;97(1):26-32. 